University of Nebraska - Lincoln

DigitalCommons@University of Nebraska - Lincoln

2019

Infertility patient-provider communication and (dis)continuity of care: An exploration of illness identity transitions

Angela L. Palmer-Wackerly

Heather L Voorhees

Sarah D’Souza

Edward Weeks

Follow this and additional works at: https://digitalcommons.unl.edu/commstudiespapers

Part of the Critical and Cultural Studies Commons, Gender, Race, Sexuality, and Ethnicity in Communication Commons, and the Other Communication Commons

This Article is brought to you for free and open access by the Communication Studies, Department of at DigitalCommons@University of Nebraska - Lincoln. It has been accepted for inclusion in Papers in Communication Studies by an authorized administrator of DigitalCommons@University of Nebraska - Lincoln. 


\title{
Infertility patient-provider communication and (dis)continuity of care: An exploration of illness identity transitions
}

\author{
Angela L. Palmer-Wackerly, ${ }^{1}$ Heather L. Voorhees, ${ }^{2}$ \\ Sarah D'Souza, ${ }^{3}$ and Edward Weeks ${ }^{4}$ \\ 1 Department of Communication Studies, The University of Nebraska-Lincoln, \\ USA \\ 2 Department of Communication Studies, The University of Nebraska-Lincoln, \\ USA \\ 3 Department of Biobehavioral Health, Pennsylvania State University, USA \\ 4 School of Communication, The Ohio State University, USA \\ Corresponding author - A.L. Palmer-Wackerly, Department of Communication Studies, \\ University of Nebraska-Lincoln, Lincoln, NE 68588, USA. \\ email apalmer-wackerly2@unl.edu
}

\begin{abstract}
Objective: To identify how and why infertility patients' communication with health care providers relates to their continuity of care within infertility treatment.

Method: A grounded theory analysis was conducted for 25 in-depth interviews across three coding phases, where we remained open to all themes present in the data, narrowed to most prominent themes, and found the connections between the themes.

Results: Based on our identified themes, we created a conceptual model that explains why infertility patients (dis)continued care with one or more clinician. Through this model, we describe two infertility identity transitions for patients:
\end{abstract}

Published in Patient Education and Counseling 102 (2019) 804-809

doi:10.1016/j.pec.2018.12.003

Copyright (C) 2018 Elsevier B.V. Used by permission.

Submitted 13 June 2018; revised 21 November 2018; accepted 2 December 2018. 
Transition 1: "Infertility as Temporary" to "Infertility as Enduring"; and Transition 2: "Infertility as Enduring" to "Infertility as Integrated."

Conclusion: The study explains how and why patients' view of their infertility affects their communication, and thus their continuity of care, with clinicians.

Practice implications: To provide patient-centered care within infertility treatment, providers can recognize how patients' view of their infertility, and thus their needs, goals, and expectations, shift throughout their infertility experience.

Keywords: Infertility, Illness identity change, Continuity of care, Patient-provider communication

\section{Introduction}

Infertility, which is defined as the inability to conceive or maintain pregnancy after attempting to conceive for 1 year, or 6 months if over the age of 35 [1], affects roughly 9 percent of the global population [2]. Treatment options vary from conventional medical interventions (e.g., medication, surgery) to more advanced assisted reproductive technologies (ART), such as inter-uterine insemination (IUI) and in vitro fertilization (IVF). The live birth rate for ART has been estimated as $49 \%$ or higher if patients continue with the recommended number of ART cycles (i.e., 3 rounds for each procedure) [3]. However, patients often opt out before completing the treatment recommended by their clinician; at fertility clinics, the drop out rate is estimated between $23 \%$ and $60 \%[4]$.

In addition to the financial, emotional, and physical stressors involved in infertility treatment, clinic-related communication issues (e.g., insufficient descriptions of fertility issues, lack of support with psychological issues) have been cited as key reasons why patients drop out of infertility treatment, especially after the first ART cycle failure $[4,5]$. As a result, researchers have turned their attention to patient-centered care (PCC), defined as care that is sensitive to patients' preferences and needs, and ensures that "patient values guide all clinic decisions" [6, p. 3]. An important factor in quality PCC is patient-provider continuity of care, which is an "enduring personal relationship between the patient and clinician that is characterized by personal trust and responsibility" [7, p. 652], which, for infertility patients, has been shown to increase tolerability of treatment and overall well-being. Despite the increased focus on PCC in infertility care, 
scholars have called for more research in three areas: (1) patient-provider continuity of care in relation to treatment outcomes; (2) theoretical development around treatment discontinuation decision-making; and (3) qualitative research to validate the scales used to explain why patients leave treatment [4, p. 662].

Additionally, patients' view of their infertility as part of their overall identity should be continually assessed to better focus care around patient values, preferences, and priorities, just as with other illness conditions (e.g., cancer) [8]. "Illness identity" develops when chronic illness forces patients to reassess their current sense of identity and future potential, thus creating new goals and expectations for themselves in an attempt to adapt [9]. As treatments continue and cycles add up, infertility can become more or less important in one's overall identity $[10,11]$, and can change the way patients and partners relate to each other in positive and negative ways [12]. Much research has focused on the significant grief and negative outcomes experienced by infertile patients as they mourn the loss of their fertile self [10], but research has also indicated that infertility identities, experiences, choices, treatments, and outcomes are unique to each individual, and that these identities and experiences change over time [13]. However, an important gap in knowledge about patients' infertility experiences is how an evolving illness identity affects the patient-provider relationship and a patient's infertility treatment preferences, including continuity of care. Thus, in the current study, we asked infertility patients to discuss positive and negative communication experiences with their clinicians in an attempt to develop a conceptual framework for infertility identity, communication, and continuity of care.

\section{Method}

\subsection{Design and theoretical framework}

The current study was approved by the Ohio State University Institutional Review Board (Protocol Number: 2011Bo280) and was part of a larger study investigating communication issues within the infertility experience. An interpretive approach was chosen to explore "the process of interaction among individuals" [[14], p. 24]-i.e., how 
patients make sense of infertility through interactions with health care providers. Because infertility is a unique medical condition (i.e., age restrictions, optional treatment, limited health insurance coverage), we used a grounded theory analysis to explain how and why infertility patients' infertility identity relates to continuity of care, since this approach is useful when seeking multiple (and possibly conflicting) views based on different experiences [15], affected by different values, finances, and goals.

\subsection{Participants}

Study participants included 25 individuals (19 women; 6 men) who had experienced infertility, as defined by the World Health Organization [1]. Among these participants were six heterosexual and two lesbian couples (see Table 1 for participant demographics).

\subsection{Data collection}

Seeking participants with diverse demographic characteristics (i.e., ethnic/racial identity, education, income), we used a purposive recruiting approach with four methods: (1) a faculty and staff newsletter at a large Midwestern university $(\mathrm{n}=13)$, a local fertility support group $(n=7)$, a publicly accessible research database $(n=3)$, and respondent-driven sampling $(n=2)$. Seeking a variety of infertility experiences, we recruited individuals who were 18-50 years old and had experienced infertility at any time in their lives. Participants chose the interview location (e.g., coffee shop, their home), with two interviews conducted over the phone for participant convenience. Interviews ranged from approximately 60 to $90 \mathrm{~min}$. For couples, separate interviews were conducted to allow each partner to freely and privately describe their experience.

Data was collected via in-depth, semi-structured interviews by the first author [16] (see Appendix A). Following the interview, participants were asked to complete a demographic questionnaire. Interviews continued until saturation was reached (i.e., no new themes appearing in the data) [15]. 
Table 1 Participant Statistics.

Characteristic

Number (\%)

\begin{tabular}{|c|c|}
\hline & $\mathrm{N}=25$ \\
\hline Age & $M=33.8(S D=4.25)$ \\
\hline \multicolumn{2}{|r|}{ 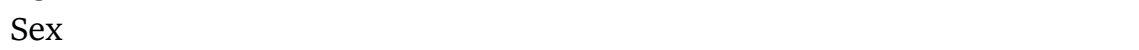 } \\
\hline Female & $19(76 \%)$ \\
\hline Male & $6(24 \%)$ \\
\hline \multicolumn{2}{|l|}{ Race } \\
\hline White & $23(92 \%)$ \\
\hline African-American & $1(4 \%)$ \\
\hline Asian/Asian-American/Pacific Islander & $1(4 \%)$ \\
\hline \multicolumn{2}{|l|}{ Education Level } \\
\hline Graduate/Professional degree & $14(56 \%)$ \\
\hline College graduate & $7(28 \%)$ \\
\hline Some college/vocational & $4(16 \%)$ \\
\hline \multicolumn{2}{|l|}{ Annual Income } \\
\hline$\$ 10,000$ to $\$ 29,999$ & $1(4 \%)$ \\
\hline$\$ 30,000$ to $\$ 49,999$ & $2(8 \%)$ \\
\hline$\$ 50,000$ to $\$ 69,999$ & $4(16 \%)$ \\
\hline$\$ 70,000$ to $\$ 89,999$ & $7(28 \%)$ \\
\hline$\$ 90,000$ to $\$ 109,999$ & $4(16 \%)$ \\
\hline$\$ 110,000$ to $\$ 129,999$ & $5(20 \%)$ \\
\hline$\$ 130,000$ to $\$ 150,000$ & $2(8 \%)$ \\
\hline \multicolumn{2}{|l|}{ Current (In)fertility Status } \\
\hline Infertile, not trying to get pregnant & $12(48 \%)$ \\
\hline Infertile, trying to get pregnant & $10(40 \%)$ \\
\hline Pregnant & $3(12 \%)$ \\
\hline \multicolumn{2}{|l|}{ Infertility Diagnosis } \\
\hline Female Factor & $19(76 \%)$ \\
\hline Unexplained & $6(24 \%)$ \\
\hline \multicolumn{2}{|l|}{ Infertility Treatment } \\
\hline IVF & $9(36 \%)$ \\
\hline IUI & $5(20 \%)$ \\
\hline Surgery & $5(20 \%)$ \\
\hline Medication Only & $3(12 \%)$ \\
\hline Embryo Adoption & $2(8 \%)$ \\
\hline Nothing & $1(4 \%)$ \\
\hline \multicolumn{2}{|l|}{ Experienced Miscarriage } \\
\hline Yes & $15(60 \%)$ \\
\hline No & $10(40 \%)$ \\
\hline
\end{tabular}




\subsection{Analysis}

As each interview was completed, the first author transcribed each one verbatim, assigned pseudonyms to interviewees, stored it on a password-protected computer, and uploaded it to Nvivo (QSR International Version 11). During initial coding, two researchers separately read through each transcript at least twice and coded units of analysis (i.e., any meaningful thought related to communication with infertility clinicians), ranging from a sentence to several paragraphs. Each researcher completed one memo per transcript, in which they summarized data, identified new ideas, and highlighted similarities with previous transcripts [14]. This process allowed us to identify patterns, new ideas, or remaining gaps, and discuss them as a team. After initial coding, two additional, independent researchers re-coded the data, based on the initial findings, to: (1) verify key findings, and (2) identify any new ideas in the data [15]. Coders met regularly for five weeks and established reliability by discussing and resolving coding discrepancies in the transcripts, and identifying any additional themes related to support and identity [15].

\section{Results}

Overall, we found that infertility patients' needs, goals, and expectations for treatment and provider communication changed as their view of their infertility identity changed. Specifically, we identified two key illness identity transitions for patients during their infertility experience, which resulted in changes in how patients approached communication with clinicians (see Figure 1). We found that regardless of sexual orientation, income, or other demographics, patients (and partners) had similar infertility identity transitions, even if infertility experiences were different. In particular, we found that when clinicians accommodated patients' infertility identity changes by meeting their changing communication needs, patients were more likely to continue care with these clinicians. When their communication needs were not met, patients were more likely to seek care elsewhere or discontinue treatment (see Table $\mathbf{2}$ for patient-provider communication recommendations). To give an in-depth account of patients' infertility 


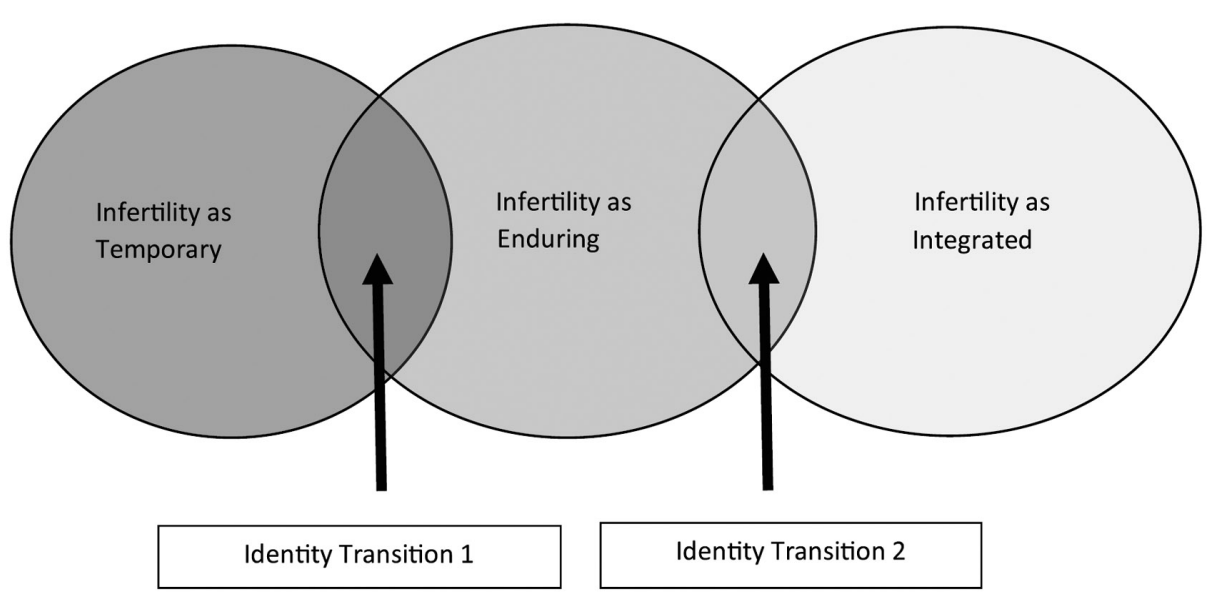

Figure 1. Conceptual Model of Changing Infertility Identity and Continuity of Care. Transitions mark shifts, where patients' infertility goals and expectations for infertility treatment and clinician communication change.

Table 2 Suggestions for Improving Infertility Continuity of Care.

When patients are viewing their "Infertility as Enduring", clinicians should:

1 Tailor each treatment recommendation to patients' specific infertility diagnosis so that patients understand why their treatment is the best current option for them.

2 Explain the limits of current medical knowledge when diagnosing 'unexplained infertility' so that patients understand that this diagnosis is valid.

3 Give patients time to ask questions and do not rush them to process complex information in decision-making or they may feel that you do not care about their personal situation.

4 Discuss all treatment options (even if not recommended) in order for patients to feel like they are an informed partner in their treatment decisions.

When patients are viewing their "Infertility as Integrated", clinicians should:

1 Acknowledge how financially, emotionally, physically, and psychologically difficult infertility treatment can be for patients and suggest coping resources.

2 Offer emotional support to patients before recommending next treatment steps in order to assess patients' current emotions about a failed treatment cycle. Emotional support needs might increase as patients continue treatment.

3 Avoid giving 'false hope' when discussing live birth rates and 'good news' by being sensitive, honest and direct, in order for patients to be 'cautiously optimistic' about treatment outcomes.

4 Provide holistic health care to infertility patients by discussing implications of infertility (and treatment) on their overall health. 
identity transitions and communication needs, we illustrate one couple's (dis)continuity of care experience below.

\subsection{Illness identity transitions}

\subsubsection{Infertility as temporary to infertility as enduring}

Patients explained that when they entered into infertility treatment, they, like their clinicians, communicated about their diagnosis, goals, and expectations as temporary. Although most patients were initially surprised by their infertility diagnosis, patients were largely confident that pregnancy would be achieved once they began treatment; thus, their infertility was viewed as a solvable medical condition. Patients may have liked or disliked their clinicians' "bedside manner," but communication challenges were minimal (or ignored) because patients and providers were aligned with meeting the same goal: pregnancy. Even if patients were uncertain and fearful about infertility treatment, they tried to adjust to the medical reality and discomfort of 'being' an infertility patient. Thus, patients initially approached their communication with clinicians by asking few (if any) procedural questions about treatment, and trusting that their clinicians were proceeding with the best care for them.

However, if patients experienced miscarriages, they learned that a live birth (as opposed to a pregnancy) was their new goal. Thus, if treatment(s) did not result in a live birth, patients began viewing infertility as a more enduring identity. In this transition, they experienced more uncertainty, stress, and strain as they coped with ongoing, and sometimes erratic, treatment side effects (e.g., enlarged cysts, hyper-stimulated ovaries, pelvic pain); repeat procedures; and preparation for those procedures, including modifying work and vacation schedules, and creating financial plans to pay for treatment (e.g., loans, savings). They began to recognize the limits of the biomedical model in treating (and explaining) their infertility, and began to doubt the controllability of their infertility. To cope, some patients began to assume more control over their treatment and communication, and wanted clinicians to take more time to answer their amassing questions and alleviate their increasing fears. If clinicians accommodated this patient role change, patients were likely to continue in their care, but if clinicians were unable to meet the need for more 
informational support, patients blamed them, the fertility clinics, and/or the medical system at large, and often switched physicians or stopped treatment. Regardless, as patients' emotional, physical, and mental health costs increased, their need to understand their particular infertility and treatment failure(s) increased. In this identity transition, patients talked about two key goals in their conversations with clinicians: wanting individualized care and treatment; and wanting to become an active partner in treatment decision-making.

For example, when Jessica, 31, and her husband Jude, 32, decided to start trying to have children, Jessica said, "We sat down at our table. .. and said, ' $\mathrm{OK}$, this is when we're going to have our baby." She began charting her ovulation and menstruation cycles, but after 6 months she could not identify a pattern. Noting how her understanding of infertility gradually changed during this transition period, Jessica said:

We didn't really know anything about infertility... so then when it didn't happen, probably like around month 5 or 6 when we were trying, I started getting really frustrated because I thought, 'I've been on birth control my whole life. This is crazy. I'm now trying to have a baby and I can't'.. . I got progressively more upset about it.

Jessica and Jude sought care from her obstetrician-gynecologist and she was put on clomid to induce ovulation, which occurred and resulted in a pregnancy, but then she had a miscarriage. Jessica said this was "devastating," as she had assumed the clomid would work-because, at that point, Jessica still saw Infertility as Temporary. Jude, also believing their infertility could be solved with the right treatment, focused on the plan: "You know, we were discouraged but we wanted to keep taking the steps that were necessary, that were in front of us at that time."

After a few more unsuccessful rounds of clomid, Jessica and Jude's obstetrician-gynecologist referred them to a local fertility clinic. Though the couple hoped to understand why they had experienced 
infertility and a miscarriage, the clinic's specialists instead diagnosed them with "unexplained infertility" and recommended several cycles of injectibles to induce ovulation in combination with IUIs, which did not result in pregnancy. As they transitioned to viewing Infertility as Enduring, the couple began to desire an official diagnosis and a more individualized treatment plan. Jessica explained, "[My doctor] wasn't doing a lot to figure out what was going wrong. I was getting frustrated." Jude expressed similar frustration with the clinic:

They don't, in my opinion, make any effort there to diagnose. They just start doing treatments cause it's probably, they make more money off of it. I don't know. So, we started doing clomid but with monitoring, we weren't having any luck and it became more and more stressful.

During this identity transition, Jessica and Jude both desired more personalized interactions with their clinicians, and wanted to be more involved in their care decisions. Inadequate communication with both clinicians left Jessica feeling:

... blamey, you know like frustrated with doctors. And, even more so when we went [to the fertility clinic]... Also, there you're a number. When you call on the phone, you have a number that is designated to you and you wait for a long time in that waiting room with lots of other people. And, you're in there for 4 min talking to your doctor and they're gone. You don't see your same doctor consistently. They rotate you between the four doctors in that practice and it just got really frustrating. I felt like nobody cares. It was just really hard.

\subsubsection{Infertility as enduring to infertility as integrated}

As patients faced the limitations of infertility treatment to achieve a live birth, those who decided to continue treatment while struggling with grief reported that emotions like anger, fear, and sadness began to surface more in conversations with their clinicians. These patients wanted clinicians to validate their grief, their treatment goals, and their emotional, physical, and financial limits. In this transition, patients also wanted to balance infertility treatment with other aspects 
of their health, including complementary alternative medicine (i.e., acupuncture, yoga), nutrition, and fitness. What made a difference for these patients were clinicians who could help them emotionally cope with their grief and present options with how to proceed, keeping their overall health in mind. Thus, patients in this second (and final) identity transition expressed wanting empathy and validation from clinicians; realistic messages of their ability to have a live birth; and holistic care for their entire person, not just their infertility.

For example, when Jessica and Jude were unable to have their questions answered or receive the personalized attention through the "funnel of treatments" they experienced at the first fertility clinic, they sought care from another local obstetrician-gynecologist with a Roman Catholic-based practice who focused in natural family planning and in-depth charting of ovulation patterns. However, that clinician also did not provide the sensitivity or empathy that Jessica and Jude desired, and "act[ed] judgey" about their preference to try IVF, "because he was Catholic."

They tried one cycle with him, and when charting did not result in a pregnancy, they sought care from another local fertility clinic-their fourth clinician in their infertility treatment experience. After 5 cycles of clomid and 5 IUIs with injectibles with past clinicians, Jessica explained that she was exhausted and wanted someone to listen to her:

I really connected with the doctor there.. . It just felt more like, instead of her being, like, dictating to me what we were going to do, I was more of an interactive participant, you know? I really liked that. I was in a really bad place mentally. We were going to you know a counselor and all that. And, we had been at it for a year and a half and having a miscarriage. And, I was like I just want to do IVF. I'm just ready to do this. I'm tired.

After a new round of consultations and tests, her new clinician diagnosed Jessica with Polycystic Ovarian Syndrome (PCOS), which affected her ability to ovulate a quality egg. Jessica was pleased that a diagnosis was finally made, that she now had a more specific picture of her reproductive health, and that her clinician also considered infertility as part of her overall health: 
I got more hopeful around the time that we had a diagnosis, like 'Ok, I can put my finger on this. I can read about it. I can you know do something'. It made me mad too, once I realized that it had implications for my future health, related to diabetes because it's connected, that it wasn't diagnosed [at the previous clinic]. Because you know, I felt even if I don't have a baby, this is still important for me to know that I have this condition because you know I need to manage my diet and exercise even more so because of it.

Jude, too, considered it a "huge relief" for Jessica to finally get an official diagnosis, but shared her frustration of what he perceived as the first clinic's lack of care for her overall health, and called them to ask why they never looked into PCOS as a diagnosis:

I was like, 'listen, I just want an explanation, why we didn't have that?' And, they're like, 'Oh, PCOS isn't really a thing, that's just a term thrown around'. So, to not disclose or to not address that, I felt was inherently reckless from the infertility standpoint, from a larger medical perspective. So, I was really, really frustrated and disappointed.

Thus, with their new clinician at their new fertility clinic, they completed their first round of IVF. Approaching these new cycles, Jude explained that it helped them to maintain a realistic perspective for treatment outcomes:

We needed to always, as a self-preservation mechanism, hope for the best with what we were trying to do, but always have a next step in mind so that we wouldn't get too much hope invested in a given cycle or effort.. . We still stood with a 15 percent chance of success, so based on our situation, that's why with the jump to IVF.

While transitioning to Infertility as Integrated, Jessica said she started to worry what IVF would "do to my body:" 
I guess I didn't think about it too much until I got hyperstimulated and then I got really frustrated. I got upset. It hurt. It's uncomfortable. You can't really walk. We drove home [2 h], I felt every single bump in the road. . . and then you start to think, 'If this doesn't work, and I'm putting my body through this, it was all for nothing' ... It's awful. It's so painful ... and [when] it didn't work, that was pretty devastating because we thought, 'Oh my gosh, like we can't do this again for awhile.'

However, her clinician called and suggested that they do another round of IVF, this time with a frozen cycle, requiring only an embryo transfer, which is "cheaper", "easier", and "not as hard on your body". Jessica felt this suggestion was made with consideration of their increasing emotional, financial, and physical strain, so they decided to complete the transfer, which resulted in a healthy, live birth. One and half years later, she and Jude were ready to try for their second child. Even though IVF was successful for the first child, they did not want to pursue it immediately for the second. Viewing Infertility as Integrated, Jessica considered their entire situation: “We don't have $\$ 4000$ just sitting around to throw at it. And, I don't really want to put my body through all of that stuff again if I don't have to."

Her clinician was supportive and suggested using Famera, an oral medication that induces ovulation, with an accompanying trigger shot to more precisely activate ovulation. Because this treatment considered her entire health identity, Jessica was open to trying it:

It's just like really similar to clomid, but people with PCOS do better on it. It doesn't thin out your lining as much. And, you don't have as many crazy side effects. It's cheap, it's pretty easy so I feel like well, we'll try it because we haven't done it before.

Thus, with empathy and validation from clinicians, realistic messages of their ability to have a live birth, and holistic care for their entire health, Jessica and Jude continued care with this clinician and remained open to their new treatment suggestions, both for a frozen IVF cycle and Famera for a current cycle. 


\section{Discussion and conclusion}

\subsection{Discussion}

The purpose of the current study was to explore how and why infertility patients' communication experiences with their clinicians connected to continuity of care. Our results confirmed previous studies that showed infertility patients value a genuine connection with their provider (i.e., sense of partnership, personableness, empathy, concern), and this patient-centered relationship improves patient well-being, anxiety and stress, and confidence in their ability to manage their symptoms $[17,18]$. Likewise, patient information needs are dynamic and depend upon the relevance, helpfulness, and trustworthiness of clinician messages [19]; however, we show in our findings that what patients perceive as relevant, helpful, and trustworthy, changes. For example, we found that patients wanted clinicians to communicate with them differently depending upon if they viewed their infertility as temporary, enduring, or integrated (see Table 2). When patients transitioned from "infertility as temporary" to "infertility as enduring", they began to doubt the clinician information that they previously received and trusted, such as the accuracy and validity of their (non)specific diagnosis (e.g., unexplained infertility); the level of clinician openness in discussing all available treatment options, especially as they began to hear of other medications and procedures from friends; and/or the level of clinician concern for patient well-being if patients felt "rushed" or "not heard". Likewise, when patients transitioned to "infertility as integrated", they needed more emotional support from clinicians than in the past because they began accepting the limitations of infertility treatment and focusing more on their overall health while imagining life beyond infertility treatment. For example, patients wanted clinicians to communicate empathy by acknowledging how stressful infertility treatments were and suggesting coping resources; avoiding giving false hope by instead being "cautiously optimistic" when communicating about live birth rates and positive pregnancy test results; and caring about patients' overall health, not just their reproductive outcomes. Thus, while our infertility identity transitions involve biographical disruption [20] and may be similar to identity transitions found in theories examining other illness identities 
$[9,21]$, infertility identity transitions are unique because unlike other illnesses, infertility is not terminal, treatment is expensive and often not covered by health insurance, and must end at some point due to age limitations.

Our study included limitations. Because this study was exploratory, our recruiting occurred via purposive sampling and largely within one large Midwestern state; however, patients sought infertility care from local and distant clinics throughout the country, which gave depth and variability to our findings. Additionally, we used single, retrospective interviews to illustrate identity transitions that occurred over time. Although this method cannot describe how participants viewed their infertility identity changes during the moments they were experiencing them, we draw from communicated narrative sense-making theory, which asserts that individuals make sense of their identities, experiences, and challenges through communication and construct a coherence to them later that might not have been clear in the actual moment $[[22]]$.

\subsection{Conclusion}

Scholars have called for more research on infertility continuity of care $[23,24]$, and we created a model grounded in our data that acknowledges the complexity of infertility as a biopsychosocial, fluid medical condition [25], where treatments often involve high uncertainty, expense, grief, and invasiveness; and continuity of care is influenced by effective patient-provider communication.

\subsection{Practice implications}

Although patient-centered care (PCC) is viewed as a key solution to patient-provider communication challenges within infertility [23], it is difficult for providers to know exactly what patients' individual emotional, psychological, and medical needs are when visits are focused on efficiency and productivity [4], and patients vary in terms of past infertility experiences, desire for biological children, communication preferences, and decision-making roles [26]. Yet, we found that when clinicians could quickly adapt their advice and communication style to patients' changing informational and emotional needs, infertility 
patients were more willing to continue treatment with that clinician. Thus, it can be helpful to understand how patients frame their infertility identity and how and why this identity may change over time. If clinicians are more aware of patients' illness identity transitions, they may be able to more readily meet patients' needs as they start to occur, understand patients' changing perspectives of their infertility and treatment, better prepare them for next steps in treatment, and more readily seek additional guidance from patients to fulfill their needs and expectations, ultimately improving patients' continuity of care.

Funding and conflicts of interest - This research did not receive any specific grant from funding agencies in the public, commercial, or not-for-profit sectors. The authors declare they have no conflict of interest.

Acknowledgments - We are grateful for the contributions made by Dr. Janice Krieger and Dr. Emily Moyer-Gusé.

\section{Appendix A. Interview Guide}

Question 1 (Warm-up): Knowledge of their fertility issue

1) Could you walk me through your infertility experience?

a What was the diagnosis?

i Female/male factor, both, or undiagnosed?

ii Primary or secondary infertility?

b What was your reaction to this diagnosis?

c What steps/procedures did you undergo after the diagnosis?

$\mathrm{d}$ What factors do you consider when thinking about fertility treatment options?

i How did these treatments affect you?

Question 2: Language associated with infertility/fertility

2) How open do you feel people in our society are to talking about infertility?

a How do you think people talk about it in our society?

b If someone had never experienced infertility, how would you describe it?

c How has this experience changed over time?

\section{Question 3: Relationships-The way needs were helped/thwarted through} communication

3) How would you describe your relationship with your doctor(s) who oversees your fertility?

a How does your doctor talk to you about your fertility issue?

i What comments or advice were helpful to you? 
ii What comments or advice were hurtful?

iii Why do you think he/she responded this way?

b How would you like your doctor to talk about fertility with you?

c What do you perceive to be the potential negatives with these changes?

\section{Question 4: Future plans with fertility}

4) In terms of communication about infertility, how do you think people should talk about it?

5) What are your current plans now in regard to your fertility? a Future plans?

6) How has your fertility experience changed you?

7) Any advice that you have for people who are dealing with infertility?

8) Anything else that you would like to add?

\section{References}

[1] World Health Organization, Multiple Definitions of Infertility, (2016) . [Internet]. (accessed 2018 Mar 1) http://www.who.int/reproductivehealth/topics/ infertility/multiple-definitions/en/

[2] J. Boivin, L. Bunting, J.A. Collins, K.G. Nygren, International estimates of infertility prevalence and treatment-seeking: potential need and demand for infertility medical care, Hum. Reprod. 22 (2007), doi:10.1093/humrep/dem299 2800-2800.

[3] J.E. Stern, M.B. Brown, B. Luke, E. Wantman, A. Lederman, S.A. Missmer, M.D. Hornstein, Calculating cumulative live-birth rates from linked cycles of assisted reproductive technology (ART): data from the Massachusetts SART CORS, Fertil. Steril. 94 (2010) 1334-1340, doi:10.1016/j. fertnstert.2009.05.052.

[4] S. Gameiro, J. Boivin, L. Peronace, C.M. Verhaak, Why do patients discontinue fertility treatment? A systematic review of reasons and predictors of discontinuation in fertility treatment, Hum. Reprod. Update 18 (2012) 652- 669, doi:10.1093/humupd/dmso31.

[5] S. Gameiro, C.M. Verhaak, J.A.M. Kremer, J. Boivin, Why we should talk about compliance with assisted reproductive technologies (ART): a systematic review and meta-analysis of ART compliance rates, Hum. Reprod. Update 19 (2013) 124-135, doi:10.1093/humupd/dmso45.

[6] Institute of Medicine, Crossing the Quality Chasm: a New Health System for the 21st Century, National Academy Press, Washington, D.C, 2001. (accessed 1 March 2018) http://www.nationalacademies.org/hmd/ /media/Files/Report $\% 20$ Files/2001/Crossing-the-Quality-Chasm/Quality $\% 20$ Chasm $\% 20$ 2001\%20\%20report\%20brief.pdf

[7] S. Gameiro, M.C. Canavarro, J. Boivin, Patient centred care in infertility health care: direct and indirect associations with wellbeing during treatment, Patient Educ. Couns. 93 (2013) 646-654, doi:10.1016/j.pec.2013.08.015. 
[8] C.L. Park, I. Zlateva, T.O. Blank, Self-identity after cancer: survivor, victim, patient, and person with cancer, J. Gen. Intern. Med. 24 (2009) 430-435, doi:10.1007/s11606-009-0993-x.

[9] K. Charmaz, The body, identity, and self: adapting to impairment, Sociol. Q. 36 (1995) 657-680.

[10] M. Kirkman, Infertile women and the narrative work of mourning: barriers to the revision of autobiographical narratives of motherhood, Narrat. Inq. 13 (2003) 243-262, doi:10.1075/ni.13.1.o9kir.

[11] E. Neter, S. Goren, Infertility centrality in the woman's identity and goal adjustment predict psychological adjustment among women in ongoing fertility treatments, Int. J. Behav. Med. 24 (2017) 880-892, doi:10.1007/ s12529-017-9693-9.

[12] L. Schmidt, B. Holstein, U. Christensen, J. Boivin, Does infertility cause marital benefit? An epidemiological study of 2250 women and men in fertility treatment, Patient Educ. Couns. 59 (2005) 244-251, doi:10.1016/j. pec.2005.07.015.

[13] A.L. Greil, K. Slauson-Blevins, J. McQuillan, The experience of infertility: a review of recent literature, Sociol. Health Illn. 32 (2010) 140-162, doi:10.1111/j.1467-9566.2009.01213.x.

[14] J.W. Creswell, C.N. Poth, Qualitative Inquiry \& Research Design: Choosing Among Approaches, fourth ed., Sage Publications, Thousand Oaks, CA, 2018.

[15] K. Charmaz, Constructing Grounded Theory: A Practical Guide Through Qualitative Analysis, 2nd ed., Sage, Thousand Oaks, CA, 2014.

[16] M.Q. Patton, Qualitative Research and Evaluation Methods, Sage Publications, Thousand Oaks, CA, 2002.

[17] S. Fox, C. Chesla, Living with chronic illness: a phenomenological study of the health effects of the patient-provider relationship, J. Am. Acad. Nurse Pract. 20 (2008) 109-117, doi:10.1111/j.1745-7599.2007.00295.x.

[18] E.K. Willer, Health-care provider compassionate love and women's infertility stressors, Commun. Monogr. 81 (2014) 407-438, doi:10.1080/03637751. 2014.940591.

[19] R.M. Epstein, Whole mind and shared mind in clinical decision-making, Patient Educ. Couns. 90 (2013) 200-206, doi:10.1016/j.pec.2012.06.035.

[20] M. Bury, Chronic illness as biographical disruption, Sociol. Health Illn. 4 (1982) 167-182, doi:10.1111/1467-9566.ep11339939.

[21] C.M. Mathieson, H.J. Stam, Renegotiating identity: cancer narratives, Sociol. Health Illn. 17 (1995) 283-306, doi:10.1111/1467-9566.ep10933316.

[22] J.K. Kellas, H.K. Horstman, Communicated narrative sense-making: understanding family narratives, storytelling, and the construction of meaning through a communicative lens, in: L.H. Turner, R. West (Eds.), The Sage Handbook of Family Communication, Sage Publications, Thousand Oaks, CA, 2015, pp. 76-90. 
[23] E.A. Dancet, T.M. D’Hooghe, F. van der Veen, P. Bossuyt, W. Sermeus, B.W. Mol, S. Repping, Patient-centered fertility treatment: what is required? Fertil. Steril. 101 (2014) 924-926, doi:10.1016/j.fertnstert.2013.12.045.

[24] E.A. Duthie, A. Cooper, J.B. Davis, K.D. Schoyer, J. Sandlow, E.Y. Strawn, K.E. Flynn, A conceptual framework for patient-centered fertility treatment, Reprod. Health. 14 (2017) 114, doi:10.1186/s12978-017-0375-5.

[25] D.A. Gerrity, A biopsychosocial theory of infertility, Fam. J. 9 (2001) 151-158, doi:10.1177/1066480701092009.

[26] M. Malin, E. Hemminki, O. Raikkonen, S. Sihvo, M.L. Perala, What do women want? Women's experiences of infertility treatment, Soc. Sci. Med. 53 (2001) 123-133, doi:10.1016/So277-9536(oo)oo317-8. 